\title{
LAW OF THE ITERATED LOGARITHM FOR SOLUTIONS OF STOCHASTIC EQUATIONS
}

UDC 519.21

\author{
D. S. BUDKOV AND S. YA. MAKHNO
}

\begin{abstract}
Strassen's law of the iterated logarithm for a solution $x(t)$ of Itô's stochastic equation is considered in the paper. We obtain a result for small times in the uniform metric and for a more general normalizing function than the classical $\sqrt{h \ln \ln \frac{1}{h}}$.
\end{abstract}

\section{INTRODUCTION}

We consider Strassen's law of the iterated logarithm for solutions of stochastic equations for small times in the uniform metric. We use a general normalizing function $\sqrt{h} \varphi_{h}$ instead of the classical one $\sqrt{h \ln \ln \frac{1}{h}}$. A. Bulinskiǔ developed in [1] an approach to study such general normalizing functions for the Wiener process as the time goes to infinity. The result obtained below allows one to study the asymptotic behavior of integral functionals of solutions of stochastic equations. In particular, Theorem 2.2 of [5] for a Wiener process $w(t)$,

$$
\varlimsup_{h \rightarrow 0} \frac{\frac{1}{h} \int_{t}^{t+h} w(s) d s-w(t)}{\sqrt{2 h \ln \ln \frac{1}{h}}}=\frac{1}{\sqrt{3}},
$$

follows from our Theorem 2 stated below.

\section{MAin RESUlts}

This section contains the definitions and statements of the main results of the paper. The proofs are given in Section 4 below on the basis of some auxiliary results obtained in Section 3 .

Let $\left(\Omega, \mathfrak{F}, \mathfrak{F}_{t}, \mathrm{P}\right)$ be a probability space equipped with a filtration $\mathfrak{F}_{t}, t \in[0,1]$. Denote by $E_{d}$ the $d$-dimensional Euclidean space with the scalar product $(\cdot, \cdot)$. Let $C([0,1], d)$ denote the space of all continuous functions on $[0,1]$ assuming values in $E_{d}$ and let $\rho(f, g)=\sup _{t \in[0,1]}|f(t)-g(t)|$ and $\mathcal{B}$ be the metric and Borel $\sigma$-algebra in this space, respectively. The symbol $I(A)$ stands for the indicator of a random event $A$ and $\bar{A}$ denotes the complement of the event $A$.

Let $x(t)$ be a solution of the stochastic equation

$$
x(t)=x_{0}+\int_{0}^{t} b(s, x(s)) d s+\int_{0}^{t} \sigma(s, x(s)) d w(s),
$$

2010 Mathematics Subject Classification. Primary 60F10, 60F17.

Key words and phrases. Stochastic equation, large deviations, law of the iterated logarithm.

The research is supported by the Foundation for Joint Scientific Researches of National Academy of Science of Ukraine and Russian Foundation for Fundamental Researches, grant \#104. 
where $b(t, x) \in E_{d}, \sigma(t, x)$ is a $d \times n$ matrix, and $w$ is an $n$-dimensional and $\mathfrak{F}_{t}$-adapted Wiener process. Put $a(t, x)=\sigma(t, x) \sigma^{*}(t, x), \sigma_{0}=\sigma\left(0, x_{0}\right)$, and $a_{0}=\sigma_{0} \sigma_{0}^{*}$, where ${ }^{*}$ is the transposition symbol.

We assume the following conditions.

(i) There exists a constant $K$ such that

$$
\left|b_{i}(t, x)\right|+\left|\sigma_{i j}(t, x)\right| \leq K
$$

for all $i=1,2, \ldots, d$ and $j=1,2, \ldots, n$, where the functions $\sigma_{i j}(t, x)$ are continuous at the point $\left(0, x_{0}\right)$.

(ii) There exists $\lambda>0$ such that $\left(a_{0} \theta, \theta\right) \geq \lambda|\theta|^{2}$ for all $\theta \in E_{d}$.

By $\Phi$ we denote the class of increasing functions $\varphi(x), x \geq 0$, such that

$$
\lim _{x \rightarrow \infty} \varphi(x)=\infty, \quad \lim _{x \rightarrow \infty} \frac{\varphi(x)}{\sqrt{x}}=0 .
$$

Let $c>1$ be a number and let $[c]$ denote its integer part. For every $\varphi \in \Phi$, we define $R^{2}(\varphi)$ as follows:

$$
R^{2}(\varphi)=\inf \left\{r>0: \sum_{n=1}^{\infty} \exp \left\{-r \frac{\varphi^{2}\left(\left[c^{n}\right]\right)}{2}\right\}<\infty\right\}
$$

We set $R^{2}(\varphi)=\infty$ if the series in (2) diverges for all finite $r$. Note that if the series in (2) converges for some $c>1$, then it converges for all $c>1$. It is proved in [1, Theorem 3] that $R=Q^{-1}$, where

$$
Q=\varliminf_{x \rightarrow \infty} \frac{\varphi(x)}{\sqrt{2 \ln \ln x}} .
$$

For an absolutely continuous function $f(t)$, put

$$
I_{0}(f)=\frac{1}{2} \int_{0}^{1}|\dot{f}(t)|^{2} d t
$$

and introduce the following classes of functions:

$$
\begin{gathered}
\mathcal{K}_{R}=\left\{f \in(C[0,1], n): f(0)=0, I_{0}(f) \leq R^{2} / 2\right\}, \\
\mathcal{H}_{R}=\left\{g(t): g(t)=\sigma_{0} f(t), f \in \mathcal{K}_{R}, t \in[0,1]\right\} .
\end{gathered}
$$

If $R=\infty$, then $\mathcal{K}_{R}=\{f \in(C[0,1], n): f(0)=0\}$. Put $\varphi_{h}=\varphi(1 / h)$ and let $x_{h}(t)=\left(x(t h)-x_{0}\right) /\left(\sqrt{h} \varphi_{h}\right)$. We derive from (11) that

$$
x(t h)=x_{0}+h \int_{0}^{t} b(s h, x(s h)) d s+\sqrt{h} \int_{0}^{t} \sigma(s h, x(s h)) d w_{h}(s)
$$

and

$$
x_{h}(t)=\frac{\sqrt{h}}{\varphi_{h}} \int_{0}^{t} b(s h, x(s h)) d s+\frac{1}{\varphi_{h}} \int_{0}^{t} \sigma(s h, x(s h)) d w_{h}(s),
$$

where $w_{h}(t)=w(t h) / \sqrt{h}$ is a Wiener process.

Theorem 2.1 provides the functional law of the iterated logarithm for a solution of the stochastic equation (11) considered for small times. This result is used in Theorem 2.3 to study the limit behavior of integral functionals of a solution.

Below are two main results of the paper.

Theorem 2.1. Let conditions (i) and (ii) hold for the coefficients of equation (1). Then, with probability one, the family of random functions $\left\{x_{h}(t)\right\}_{h>0}$ is relatively compact in $C([0,1], d)$ and the set of limit points of this family coincides with $\mathcal{H}_{R}$. 
The properties of continuous mappings together with Theorem 2.1 imply the following result concerning the limit points of $F\left(x_{h}\right)$.

Corollary 2.2. Let $F(\cdot)$ be a continuous on $C([0,1], d)$ functional. Then, with probability one, the set of limit points of $F\left(x_{h}\right)$ coincides with $F\left(\mathcal{H}_{R}\right)$.

Consider the following family of stochastic processes:

$$
\xi_{h}(t)=\frac{\frac{1}{h} \int_{0}^{t h} x(s) d s-t x_{0}}{\sqrt{h} \varphi_{h}}, \quad t \in[0,1] .
$$

Theorem 2.3. With probability one, the family of random functions $\left\{\xi_{h}(t)\right\}_{h>0}$ is relatively compact in $C([0,1], d)$ and the set of its limit points coincides with

$$
\widetilde{\mathcal{H}}_{R}=\left\{\tilde{g}(t)=\int_{0}^{t} g(s) d s: g(s)=\sigma_{0} f(s), f \in \mathcal{K}_{R}, t \in[0,1]\right\} .
$$

Corollary 2.4. For $d=1$,

$$
\begin{aligned}
\varlimsup_{h \rightarrow 0} \frac{\frac{1}{h} \int_{0}^{h} x(s) d s-t x_{0}}{\sqrt{h} \varphi_{h}} & =\frac{\left|\sigma_{0}\right| R}{\sqrt{3}}, \\
\varliminf_{h \rightarrow 0} \frac{\frac{1}{h} \int_{0}^{h} x(s) d s-t x_{0}}{\sqrt{h} \varphi_{h}} & =-\frac{\left|\sigma_{0}\right| R}{\sqrt{3}} .
\end{aligned}
$$

\section{Auxiliary Results}

In this section, we prove some auxiliary results needed in the proof of the main results of this paper.

We say that the large deviations principle holds for a family of probability measures $\left\{\mathrm{P}_{h}\right\}_{h>0}$ defined in $(C([0,1], d), \mathcal{B})$ with a normalizing function $\varphi_{h}^{2}$ and a rate functional $I(f)$ if

1) for all open sets $G \subset \mathcal{B}$,

$$
\varliminf_{h \rightarrow 0} \varphi_{h}^{-2} \ln \mathrm{P}_{h}(G) \geq-\inf \{I(f) ; f \in G\}
$$

2) for all closed sets $U \subset \mathcal{B}$,

$$
\varlimsup_{h \rightarrow 0} \varphi_{h}^{-2} \ln \mathrm{P}_{h}(U) \leq-\inf \{I(f) ; f \in U\} ;
$$

3) for all $a<\infty$, the set $\{\psi: I(\psi) \leq a\}$ is compact;

see [3, Chapter 3].

Lemma 3.1. Let conditions (i) and (ii) hold for the coefficients of equation (11). Then the large deviations principle holds for a family of probability measures $\left\{\mathrm{P}_{h}\right\}_{h>0}$ as $h \downarrow 0$ with the normalizing function $\varphi_{h} \in \Phi$ and the following rate functional:

$$
I(f)= \begin{cases}\frac{1}{2} \int_{0}^{1}\left(a_{0}^{-1} \dot{f}(t), \dot{f}(t)\right) d t, & \text { if } f(t) \text { is absolutely continuous and } f(0)=0 \\ +\infty, & \text { otherwise, }\end{cases}
$$

where $\mathrm{P}_{h}(A)=\mathrm{P}\left\{x_{h}(\cdot) \in A\right\}$ for $A \in \mathcal{B}$.

Proof. We rewrite representation (44) for the process $x_{h}(t)$ and obtain the following equation:

where

$$
x_{h}(t)=\int_{0}^{t} b_{h}\left(s, x_{h}(s)\right) d s+\frac{1}{\varphi_{h}} \int_{0}^{t} \sigma_{h}\left(h, x_{h}(s)\right) d w_{h}(s),
$$

$$
b_{h}(s, x)=\sqrt{h} \varphi_{h}^{-1} b\left(s h, x \sqrt{h} \varphi_{h}+x_{0}\right) \quad \text { and } \quad \sigma_{h}(s, x)=\sigma\left(s h, x \sqrt{h} \varphi_{h}+x_{0}\right) .
$$


Put $a_{h}(s, x)=\sigma_{h}(s, x) \sigma_{h}^{*}(s, x)$. Under condition (i),

$$
\lim _{h \rightarrow 0} b_{h}(s, x)=0 \quad \text { and } \quad \lim _{h \rightarrow 0} a_{h}(s, x)=a_{0}
$$

uniformly in $(s, x)$ belonging to an arbitrary compact set. Thus the proof is completed by [2, Theorem 3.2.1 and Corollary 2].

The following result is a multidimensional analogue of a corollary to Theorem 5 in Section 2 of Chapter 3 in [7]. The changes in the proof are obvious.

Lemma 3.2. Let $w(t)$ be an $n$-dimensional Wiener process, $f(t)$ be a $d \times n$ matrix function whose entries are bounded by a constant $K$. We also assume that $f(t)$ is $\mathfrak{F}_{t^{-}}$ adapted. Then

$$
\mathrm{P}\left\{\sup _{t \in[0,1]}\left|\int_{0}^{t} f(s) d w(s)\right| \geq \delta\right\} \leq 2 d \exp \left\{-\frac{\delta^{2}}{2 n K^{2}}\right\}
$$

for all $\delta>0$.

Lemma 3.3. Assume that conditions (i) and (ii) hold. Then, for every $\delta>0$, there exists $h_{0}$ such that

$$
\mathrm{P}\left\{\sup _{t \in[0,1]}\left|x(t h)-x_{0}\right| \geq \delta\right\} \leq 2 d \exp \left\{-\frac{\delta^{2}}{8 d n K^{2} h}\right\}
$$

for all $h<h_{0}$.

Proof. It follows from (3) that

$$
\begin{aligned}
\mathrm{P}\left\{\sup _{t \in[0,1]}\left|x(t h)-x_{0}\right| \geq \delta\right\} \leq & \mathrm{P}\left\{h \sup _{t \in[0,1]}\left|\int_{0}^{t} b(s h, x(s h)) d s\right| \geq \frac{\delta}{2}\right\} \\
& +\mathrm{P}\left\{\sqrt{h} \sup _{t \in[0,1]}\left|\int_{0}^{t} \sigma(s h, x(s h)) d w_{h}(s)\right| \geq \frac{\delta}{2}\right\} .
\end{aligned}
$$

Since the coefficient $b(t, x)$ is bounded, the first term on the right hand side of (7) vanishes if $h$ is sufficiently small. The second term on the right hand side of (7) is estimated by Lemma 3.2. The proof is completed.

Lemma 3.4. Assume that conditions (i) and (ii) hold. For all $L>0$ and $\delta>0$, there exists $h_{0}$ such that

$$
\mathrm{P}\left\{\sup _{t \in[0,1]}\left|\int_{0}^{t}\left(\sigma(s h, x(s h))-\sigma\left(0, x_{0}\right)\right) d w(s)\right| \geq \delta \varphi_{h}\right\} \leq 4 d \exp \left\{-L \varphi_{h}^{2}\right\}
$$

for all $h<h_{0}$.

Proof. According to condition (i), the functions $\sigma_{i j}(t, x)$ are continuous at the point $\left(0, x_{0}\right)$. Thus, given $\varepsilon>0$, there exists $\gamma>0$ such that

$$
\left|\sigma(s, x)-\sigma\left(0, x_{0}\right)\right|<\varepsilon
$$

for $0 \leq s<\gamma$ and $\left|x-x_{0}\right|<\gamma$.

Further,

$$
\mathrm{P}\left\{\sup _{t \in[0,1]}\left|\int_{0}^{t}\left(\sigma(s h, x(s h))-\sigma\left(0, x_{0}\right)\right) d w(s)\right| \geq \delta \varphi_{h}\right\}=I_{1}+I_{2},
$$


where

$$
\begin{aligned}
& I_{1}=\mathrm{P}\left\{\sup _{t \in[0,1]}\left|\int_{0}^{t}\left(\sigma(s h, x(s h))-\sigma\left(0, x_{0}\right)\right) d w(s)\right| \geq \delta \varphi_{h}, \sup _{t \in[0,1]}\left|x(t h)-x_{0}\right| \geq \gamma\right\}, \\
& I_{2}=\mathrm{P}\left\{\sup _{t \in[0,1]}\left|\int_{0}^{t}\left(\sigma(s h, x(s h))-\sigma\left(0, x_{0}\right)\right) d w(s)\right| \geq \delta \varphi_{h}, \sup _{t \in[0,1]}\left|x(t h)-x_{0}\right|<\gamma\right\} .
\end{aligned}
$$

The term $I_{1}$ in (10) is estimated by inequality (6) as follows:

$$
I_{1} \leq \mathrm{P}\left\{\sup _{t \in[0,1]}\left|x(t h)-x_{0}\right| \geq \gamma\right\} \leq 2 d \exp \left\{-\frac{\gamma^{2}}{8 d n K^{2} h}\right\} .
$$

Since $h \varphi_{h}^{2} \rightarrow 0$, one can find $h_{1}$ for a given $L>0$ such that

$$
\frac{\gamma^{2}}{8 n d K^{2} h \varphi_{h}^{2}} \geq L
$$

for $h<h_{1}$. This together with (11) implies that

$$
I_{1} \leq 2 d \exp \left\{-L \varphi_{h}^{2}\right\} \text {. }
$$

To estimate the term $I_{2}$, we use (9) and bound (5). Then

$$
I_{2} \leq 2 d \exp \left\{-\varphi_{h}^{2}\left(\frac{\delta^{2}}{8 n d \varepsilon^{2} h}\right)\right\}
$$

For all $L>0$, there exists $h_{2}$ such that the expression in the parentheses on the right hand side of (13) is greater than or equal to $L$ if $h<h_{2}$. In this case, (14) implies that

$$
I_{2} \leq 2 d \exp \left\{-L \varphi_{h}^{2}\right\} \text {. }
$$

Putting $h_{0}=h_{1} \wedge h_{2}$ in (12) and (14) we prove (8). The proof is completed.

Lemma 3.5. For all functions $f \in \mathcal{K}_{R}$ and all $\delta>0$, there exist $\gamma>0$ and $h_{0}>0$ such that

$$
\mathrm{P}\left\{\sup _{t \in[0,1]}\left|\int_{0}^{t}\left(\sigma(s h, x(s h))-\sigma_{0}\right) \dot{f}(s) d s\right| \geq \delta, \sup _{t \in[0,1]}\left|x(t h)-x_{0}\right|<\gamma\right\}=0
$$

for all $h<h_{0}$.

Proof. Using the $\gamma$ specified for (9) we obtain

$$
\delta^{2} \leq \sup _{t \in[0,1]}\left|\int_{0}^{t}\left(\sigma(s h, x(s h))-\sigma_{0}\right) \dot{f}(s) d s\right|^{2} \leq \sup _{t \in[0,1]} \varepsilon^{2} \int_{0}^{t}|\dot{f}(s)|^{2} d s \leq \varepsilon^{2} R^{2} .
$$

The random event in the braces on the left hand side of (15) has the probability zero if $\varepsilon<\delta / R$ in (9). The lemma is proved.

Denote by $H_{1}([0,1], n)$ the space of absolutely continuous functions $f(t) \in E_{n}$ such that

$$
\int_{0}^{1}|\dot{f}(s)|^{2} d s<\infty .
$$

Lemma 3.6. The functional $I(f)$ defined in Lemma 3.1 admits the following representation:

$$
I(f)=\inf _{g \in H_{1}([0,1], n)}\left\{I_{0}(g): f(t)=\sigma_{0} g(t), g(0)=0\right\}
$$

(we agree that the infimum of an empty set is equal to $+\infty$ ). 
Proof. Consider the following stochastic processes $\xi_{\varepsilon}(t)=\varepsilon w(t)$ and $\eta_{\varepsilon}(t)=\varepsilon \sigma_{0} w(t)$. It is well known that $I_{0}(f)$ and $I(f)$ are rate functionals for the processes $\xi_{\varepsilon}(t)$ and $\eta_{\varepsilon}(t)$, respectively (see [3, Theorem 5.3.1]). On the other hand, $\eta_{\varepsilon}(t)=F\left(\xi_{\varepsilon}(t)\right.$ ), where

$$
F(x)=\sigma_{0} x .
$$

Representation (16) follows from the contraction principle; see [3, Theorem 3.3.1]. The lemma is proved.

The following result for $\varphi_{h}=\sqrt{2 \ln \ln \frac{1}{h}}$ is Theorem 1 of [4]. The proof for the case considered in the current paper is given in [6, Theorem 2].

Lemma 3.7. With probability one, the set of limit points, as $h \rightarrow 0$, of the family

$$
\left\{\frac{w(t h)}{\sqrt{h} \varphi_{h}}\right\}
$$

coincides with the set $\mathcal{K}_{R}$.

\section{Proof of the main Results}

In this section, we prove the main results of the paper.

Proof of Theorem 2.1. We split the proof of this result into the three standard steps. Put

$$
\mathcal{L}_{R}=\left\{f: f(0)=0, I(f) \leq \frac{R^{2}}{2}\right\} .
$$

Lemma 3.6 implies that if $f \in \mathcal{L}_{R}$, then a function $g \in \mathcal{K}_{R}$ exists such that $f(t)=\sigma_{0} g(t)$, that is, $f \in \mathcal{H}_{R}$, whence

$$
\mathcal{L}_{R} \subseteq \mathcal{H}_{R}
$$

Set $h_{k}=\left[c^{k}\right]^{-1}$ and $z_{k}(t)=x_{h_{k}}(t)$, where $c>1$ is a given number.

Step 1. We show that, for all $c>1, \delta>0$, and $R^{2}<\infty$, an integer number $k_{0}>0$ exists such that

$$
\rho\left(z_{k}, \mathcal{H}_{R}\right)<\delta
$$

with probability one for all $k>k_{0}$. Put $B_{\delta}=\left\{f: \rho\left(f, \mathcal{L}_{R}\right) \geq \delta\right\}$. Note that a number $\delta>0$ exists such that

$$
\inf _{f \in B_{\delta}} I(f) \geq \frac{R^{2}(\varphi)}{2}+\delta .
$$

Since the large deviation principle holds for $z_{h}(t)$,

$$
\ln \mathrm{P}\left\{z_{k} \in B_{\delta}\right\} \leq-\varphi^{2}\left(\left[c^{k}\right]\right) \inf _{f \in B_{\delta}} I(f)
$$

for a closed set $B_{\delta}$ and for sufficiently large $k$. Then

$$
\mathrm{P}\left\{z_{k} \in B_{\delta}\right\} \leq \exp \left\{-\varphi^{2}\left(\left[c^{k}\right]\right)\left(\frac{R^{2}(\varphi)}{2}+\delta\right)\right\} .
$$

Recalling the definition of the number $R^{2}(\varphi)$ we deduce from the Borel-Cantelli lemma that, with probability one,

$$
\rho\left(z_{k}, \mathcal{L}_{R}\right)<\delta
$$

for sufficiently large $k$. Now (17) and (19) imply (18).

Step 2. Now we show that each limit point of the sequence $x_{h}$ belongs to $\mathcal{H}_{R}$. This is already proved for $h_{k}=\left[c^{k}\right]^{-1}$ in Step 1 . Let $h \in\left[h_{k+1}, h_{k}\right]$. 
Since $\sqrt{h} \varphi(h)$ is an increasing function,

$$
\frac{1}{\sqrt{h} \varphi(h)}=\frac{\alpha(h, k)}{\sqrt{h_{k}} \varphi_{h_{k}}}+\frac{\beta(h, k)}{\sqrt{h_{k+1}} \varphi_{h_{k+1}}},
$$

where $\alpha(h, k)>0$ and $\beta(h, k)>0$ are such that $\alpha(h, k)+\beta(h, k)=1$. Let

$$
\hat{x}_{h, k}(t)=\alpha(h, k) z_{k}(t)+\beta(h, k) z_{k+1}(t) .
$$

Then $\hat{x}_{h, k} \in\left\{f: \rho\left(f, \mathcal{H}_{R}\right)<\delta\right\}$ for sufficiently large $k$, since $\alpha f(t)+\beta g(t) \in \mathcal{L}_{R}$ for $\alpha>0$ and $\beta>0$ such that $\alpha+\beta=1$, provided that $f(t), g(t) \in \mathcal{L}_{R}$.

The goal of Step 2 is achieved if we show that, for all $\delta>0$, there exist a number $c_{\delta}>1$ and an integer $k_{0}$ such that

$$
\sup _{t \in[0,1], h \in\left[h_{k+1}, h_{k}\right]}\left|x_{h}(t)-\hat{x}_{h, k}(t)\right| \leq \delta
$$

for all $k>k_{0}$ and $c>c_{\delta}$. Recalling the definition of $z_{k}(t)$ and accounting equality (20) we obtain

$$
x_{h}(t)=z_{k}\left(t \frac{h}{h_{k}}\right) \frac{\sqrt{h_{k}} \varphi_{h_{k}}}{\sqrt{h} \varphi_{h}}=z_{k}\left(t \frac{h}{h_{k}}\right) \alpha(h, k)+z_{k+1}\left(t \frac{h}{h_{k+1}}\right) \beta(h, k) .
$$

Then

$$
\begin{aligned}
\sup _{t \in[0,1], h \in\left[h_{k+1}, h_{k}\right]}\left|x_{h}(t)-\hat{x}_{h, k}(t)\right| \leq & \sup _{t \in[0,1], s \in[t / c, t]}\left|z_{k}(t)-z_{k}(s)\right| \\
& +\sup _{t \in[0,1], s \in[t, t c \wedge 1]}\left|z_{k+1}(t)-z_{k+1}(s)\right| .
\end{aligned}
$$

Now we derive the inequality

$$
\begin{aligned}
& \mathrm{P}\left\{\sup _{t \in[0,1], h \in\left[h_{k+1}, h_{k}\right]}\left|x_{h}(t)-\hat{x}_{h, k}(t)\right| \geq \delta\right\} \\
& \leq \mathrm{P}\left\{\sup _{t \in[0,1], s \in[t / c, t]}\left|z_{k}(t)-z_{k}(s)\right| \geq \frac{\delta}{2}\right\} \\
& \quad+\mathrm{P}\left\{\sup _{t \in[0,1], s \in[t, t c \wedge 1]}\left|z_{k+1}(t)-z_{k+1}(s)\right| \geq \frac{\delta}{2}\right\} .
\end{aligned}
$$

We apply the large deviation principle to estimate the terms on the right hand side of the latter relation. Let

$$
\begin{aligned}
& Q_{\delta}=\{f \in C([0,1], n): f(t) \text { is absolutely continuous } \\
& \text { and such that } \left.\sup _{t \in[0,1], s \in[t / c, t]}|f(t)-f(s)| \geq \frac{\delta}{2}\right\} .
\end{aligned}
$$

Note that the set $Q_{\delta}, \delta>0$, is closed. Applying the Cauchy-Schwarz inequality, we prove that

$$
\frac{\delta^{2}}{4} \leq|f(t)-f(s)|^{2} \leq\left|\int_{s}^{t} \dot{f}(v) d v\right|^{2} \leq(t-s) \int_{s}^{t}|\dot{f}(v)|^{2} d v \leq \frac{2(c-1)}{\lambda} I(f),
$$

whence

$$
I(f) \geq \frac{\lambda \delta^{2}}{8(c-1)}
$$


for $f \in Q_{\delta}$. If $c_{\delta}=1+\lambda \delta^{2} /\left(4 R^{2}\right)$, then

$$
\mathrm{P}\left\{\sup _{t \in[0,1], s \in[t / c, t]}\left|z_{k}(t)-z_{k}(s)\right| \geq \frac{\delta}{2}\right\}=\mathrm{P}\left\{z_{k} \in Q_{\delta}\right\} \leq \exp \left\{-\frac{R^{2} \varphi_{h_{k}}^{2}}{2}\right\}
$$

for $c>c_{\delta}$.

Reasoning similarly we prove that

$$
\mathrm{P}\left\{\sup _{t \in[0,1], s \in[t, t c \wedge 1]}\left|z_{k+1}(t)-z_{k+1}(s)\right| \geq \frac{\delta}{2}\right\} \leq \exp \left\{-\frac{R^{2} \varphi_{h_{k}}^{2}}{2}\right\}
$$

for the same $c$. Now we obtain inequality (21) from relations (22) and (23) and from the Borel-Cantelli lemma.

Step 3. We show, for an arbitrary function $g(t)=\sigma_{0} f(t) \in \mathcal{H}_{R}$, that a subsequence exists such that $x_{h_{k}} \rightarrow g$ almost surely. Consider the following random events:

$$
C_{h}=\left\{\sup _{t \in[0,1]}\left|\frac{w_{h}(t)}{\varphi_{h}}-f(t)\right| \leq \varepsilon\right\}, \quad D_{h}=\left\{\sup _{t \in[0,1]}\left|x_{h}(t)-g(t)\right|<\delta\right\} .
$$

It is easy to see that

$$
C_{h}=\left(C_{h} \cap D_{h}\right) \cup\left(C_{h} \cap \bar{D}_{h}\right) \subset D_{h} \cup\left(C_{h} \cap \bar{D}_{h}\right) .
$$

By Lemma 3.7, the events $C_{h}$ occur infinitely often (i.o.) for whatever $\varepsilon>0$. Thus

$$
1=\mathrm{P}\left\{C_{h} \text { i.o. }\right\} \leq \mathrm{P}\left\{D_{h} \text { i.o. }\right\}+\mathrm{P}\left\{C_{h} \cap \bar{D}_{h} \text { i.o. }\right\} .
$$

Now we show that a sequence $h_{k}$ exists such that the second term on the right hand side of inequality (24) equals zero. We have

$$
\begin{aligned}
\mathrm{P}\left\{C_{h} \cap \bar{D}_{h}\right\}= & \mathrm{P}\left\{\sup _{t \in[0,1]} \mid \frac{\sqrt{h}}{\varphi_{h}} \int_{0}^{t} b(s h, x(s h)) d s\right. \\
& +\int_{0}^{t} \sigma(s h, x(s h)) d\left(\frac{w_{h}(s)}{\varphi_{h}}-f(s)\right) \\
& +\int_{0}^{t}\left(\sigma\left(s h, x(s h)-\sigma_{0}\right)\right) \dot{f}(s) d s \mid \geq \delta ; \\
\leq & \mathrm{P}\left\{\sup _{\left.t \in[0,1]\left|\frac{\sqrt{h}}{\varphi_{h}} \int_{0}^{t} b(s h, x(s h)) d s\right| \geq \frac{\delta}{3}\right\}}\left|\frac{w_{h}(t)}{\varphi_{h}}-f(t)\right| \leq \varepsilon\right\} \\
& +\mathrm{P}\left\{\sup _{t \in[0,1]}\left|\int_{0}^{t}\left(\sigma(s h, x(s h))-\sigma_{0}\right) \dot{f}(s) d s\right| \geq \frac{\delta}{3}\right\} \\
& +\mathrm{P}\left\{\sup _{t \in[0,1]}\left|\int_{0}^{t} \sigma(s h, x(s h)) d\left(\frac{w_{h}(s)}{\varphi_{h}}-f(s)\right)\right| \geq \frac{\delta}{3} ;\right. \\
= & P \\
1 & +P_{2}+P_{3} .
\end{aligned}
$$


Since $b(t, x)$ is a bounded function, there exists $h_{0}$ such that

$$
P_{1}=\mathrm{P}\left\{\sup _{t \in[0,1]}\left|\frac{\sqrt{h}}{\varphi_{h}} \int_{0}^{t} b(s h, x(s h)) d s\right| \geq \frac{\delta}{3}\right\}=0
$$

for all $0<h<h_{0}$. Further,

$$
\begin{aligned}
P_{2}= & \mathrm{P}\left\{\sup _{t \in[0,1]}\left|\int_{0}^{t}\left(\sigma(s h, x(s h))-\sigma\left(0, x_{0}\right)\right) \dot{f}(s) d s\right| \geq \frac{\delta}{3}\right\} \\
= & \mathrm{P}\left\{\sup _{t \in[0,1]}\left|\int_{0}^{t}\left(\sigma(s h, x(s h))-\sigma\left(0, x_{0}\right)\right) \dot{f}(s) d s\right| \geq \frac{\delta}{3} ; \sup _{t \in[0,1]}\left|x(h t)-x_{0}\right| \geq \gamma\right\} \\
& +\mathrm{P}\left\{\sup _{t \in[0,1]}\left|\int_{0}^{t}\left(\sigma(s h, x(s h))-\sigma\left(0, x_{0}\right)\right) \dot{f}(s) d s\right| \geq \frac{\delta}{3} ; \sup _{t \in[0,1]}\left|x(h t)-x_{0}\right|<\gamma\right\} \\
= & P_{4}+P_{5} .
\end{aligned}
$$

We pick up a number $\gamma$ such that $P_{5}=0$ by Lemma 3.5. Now Lemma 3.3 with such a number $\gamma$ yields

$$
P_{4} \leq \mathrm{P}\left\{\sup _{t \in[0,1]}\left|x(h t)-x_{0}\right| \geq \gamma\right\} \leq 2 d \exp \left\{-\frac{\gamma^{2}}{8 n K^{2} d h}\right\} .
$$

Choosing $h_{0}$ in such a way that

$$
-\frac{\gamma^{2}}{8 n K^{2} d h} \leq-R^{2} \varphi_{h}^{2}
$$

for $h<h_{0}$ we get

$$
P_{2} \leq 2 d \exp \left\{-\frac{R^{2} \varphi_{h}^{2}}{2}\right\} .
$$

Now we estimate the probability $P_{3}$ in (25). Let

$$
G_{h}=\exp \left\{\varphi_{h} \int_{0}^{1} \dot{f}(s) d w_{h}(s)-\frac{\varphi_{h}^{2}}{2} \int_{0}^{1}|\dot{f}(s)|^{2} d s\right\}
$$

and let $\widetilde{\mathrm{P}}_{h}$ be a probability measure on $(\Omega, F)$ possessing a density which we denote by $G_{h}$. By Girsanov's theorem, $\widetilde{w}_{h}(t)=w_{h}(t)-\varphi_{h} f(t)$ is a Wiener process on $\left(\Omega, \mathcal{F}, \widetilde{\mathrm{P}}_{h}\right)$.

Put

$$
S_{h}=\left\{\sup _{t \in[0,1]}\left|\int_{0}^{t} \sigma(s h, x(s h)) d \widetilde{w}_{h}(s)\right| \geq \frac{\delta \varphi_{h}}{3} ; \sup _{t \in[0,1]}\left|\widetilde{w}_{h}(t)\right| \leq \varepsilon \varphi_{h}\right\} .
$$

By $\widetilde{\mathrm{E}}_{h}$ we denote the expectation with respect to the measure $\widetilde{\mathrm{P}}_{h}$. Then

$$
P_{3}=\widetilde{\mathrm{E}}_{h} I\left(S_{h}\right) G_{h}^{-1} \leq\left(\widetilde{\mathrm{P}}_{h}\left(S_{h}\right)\right)^{\frac{1}{2}}\left(\widetilde{\mathrm{E}}_{h} G_{h}^{-2}\right)^{\frac{1}{2}} .
$$

Further,

$$
\begin{aligned}
\widetilde{\mathrm{P}}_{h}\left(S_{h}\right) \leq & \widetilde{\mathrm{P}}_{h}\left\{\sup _{t \in[0,1]} \mid \int_{0}^{t}\left(\sigma\left(s h, x(s h)-\sigma_{0}\right) d \widetilde{w}_{h}(s) \mid \geq \frac{\delta \varphi_{h}}{6}\right\}\right. \\
& +\widetilde{\mathrm{P}}_{h}\left\{\sup _{t \in[0,1]}\left|\sigma_{0} \widetilde{w}_{h}(s)\right| \geq \frac{\delta \varphi_{h}}{6} ; \sup _{t \in[0,1]}\left|\widetilde{w}_{h}(t)\right| \leq \varepsilon \varphi_{h}\right\} .
\end{aligned}
$$


Let $\alpha=\sum_{i j}\left(\sigma_{0}\right)_{i j}^{2}$. We choose $\varepsilon<\delta /(6 \alpha)$. Then the second term on the right hand side of (29) equals zero. We estimate the first term in (29) by Lemma 3.4. Therefore, for all $L>0$ and $\delta>0$, there exists $h_{0}$ such that

$$
\widetilde{\mathrm{P}}_{h}\left(S_{h}\right) \leq 4 d \exp \left\{-L \varphi_{h}^{2}\right\}
$$

for $h<h_{0}$. Since $f \in \mathcal{K}_{R}$, we obtain

$$
\begin{aligned}
& \widetilde{\mathrm{E}}_{h}\left(G_{h}^{-1}\right)^{2}=\widetilde{\mathrm{E}}_{h}\left(\exp \left\{-2 \varphi_{h} \int_{0}^{1} \dot{f}(s) d w_{h}(s)+\varphi_{h}^{2} \int_{0}^{1}|\dot{f}(s)|^{2} d s\right\}\right) \\
& =\widetilde{\mathrm{E}}_{h}\left(\exp \left\{-2 \varphi_{h} \int_{0}^{1} \dot{f}(s) d w_{h}(s)-2 \varphi_{h}^{2} \int_{0}^{1}|\dot{f}(s)|^{2} d s\right\}\right) \\
& \times \exp \left\{3 \varphi_{h}^{2} \int_{0}^{1}|\dot{f}(s)|^{2} d s\right\} \\
& \leq \exp \left\{3 R^{2} \varphi_{h}^{2}\right\} .
\end{aligned}
$$

Relations (30) and (31) yield

$$
P_{3} \leq \sqrt{4 d} \exp \left\{-\frac{\varphi_{h}^{2}}{2}\left(L-3 R^{2}\right)\right\}
$$

where the probability $P_{3}$ is defined by (25). Choosing $L=4 R^{2}$ in this inequality, we get

$$
P_{3} \leq \exp \left\{-\frac{R^{2} \varphi_{h}^{2}}{2}\right\}
$$

Turning back to relations (25), we note by (26), (27), and (32) that a number $h_{0}$ exists such that

$$
\mathrm{P}\left\{C_{h} \cap \bar{D}_{h}\right\} \leq(2 d+1) \exp \left\{-\frac{R^{2} \varphi_{h}^{2}}{2}\right\}
$$

for $h<h_{0}$. Thus a number $c>1$ exists such that

$$
\sum_{k} P\left\{C_{h_{k}} \cap \bar{D}_{h_{k}}\right\} \leq(2 d+1) \sum_{k} \exp \left\{-\frac{R^{2} \varphi^{2}\left(\left[c^{k}\right]\right)}{2}\right\}
$$

for the sequence $h_{k}=\left[c^{k}\right]^{-1}$.

Recalling the definition of $R$, we conclude that the latter series converges. Using the Borel-Cantelli lemma, we conclude that $\mathrm{P}\left\{C_{h_{k}} \cap \bar{D}_{h_{k}}\right.$ i.o. $\}=0$ for all $\delta>0$. Inequality (24) implies that

$$
\mathrm{P}\left\{D_{h_{k}}\right\}=\mathrm{P}\left\{\sup _{t \in[0,1]}\left|x_{h_{k}}(t)-g(t)\right|<\delta \text { i.o. }\right\}=1
$$

for all $\delta>0$. The theorem is proved.

Proof of Theorem 2.3. It remains to apply Corollary 2.2 and choose the functional

$$
F(x(\cdot))=\int_{0}^{t} x(s) d s .
$$

Proof of Corollary 2.4. If $\tilde{g} \in \widetilde{\mathcal{H}}_{R}$, then

$$
\begin{aligned}
\tilde{g}^{2}(1) & =\sigma_{0}^{2}\left(\int_{0}^{1} \int_{0}^{t} \dot{f}(s) d s d t\right)^{2}=\sigma_{0}^{2}\left(\int_{0}^{1}(1-s) \dot{f}(s) d s\right)^{2} \\
& \leq \sigma_{0}^{2} \int_{0}^{1}(1-s)^{2} d s \int_{0}^{1}(\dot{f}(s))^{2} d s=\frac{\sigma_{0}^{2}}{3} \int_{0}^{1}(\dot{f}(s))^{2} d s \leq \frac{\sigma_{0}^{2} R^{2}}{3} .
\end{aligned}
$$


This implies that

$$
-\frac{\left|\sigma_{0}\right| R}{\sqrt{3}} \leq \tilde{g}(1) \leq \frac{\left|\sigma_{0}\right| R}{\sqrt{3}} .
$$

Note that the upper and lower bounds are attained at the functions

$$
f_{1}(t)=\frac{\left|\sigma_{0}\right| R \sqrt{3}}{\sigma_{0}}\left(t-\frac{t^{2}}{2}\right)
$$

and

respectively.

$$
f_{2}(t)=-f_{1}(t)
$$

\section{Acknowledgment}

The authors are grateful to a reviewer for a careful reading of the manuscript and comments allowing us to improve the style of the presentation of our results.

\section{BIBLIOGRAPHY}

1. A. V. Bulinskiǔ, A new variant of the functional law of the iterated logarithm, Teor. Veroyatnost. Primenen. 25 (1980), 502-512; English transl. in Theory Probab. Appl. 25 (1981), 493-503. MR582580 (82f:60066)

2. A. D. Wentzell, Limit Theorems on Large Deviations for Markov Stochastic Processes, Nauka, Moscow, 1986; English transl., Kluwer Academic Publishers Group, Dordrecht, 1990. MR $1135113(92 \mathrm{i}: 60054)$

3. A. D. Wentzell and M. I. Freidlin, Random Perturbations of Dynamical Systems, Nauka, Moscow, 1979; English transl., Springer-Verlag, New York, 1984. MR722136 (85a:60064)

4. N. Gantert, An inversion of Strassen's law of the iterated logarithm for small time, Ann. Probab. 21 (1993), 1045-1049. MR1217579 (94d:60130)

5. D. Khoshnevisan, Exact rates of convergence to Brownian local time, Ann. Probab. 22 (1994), 1295-1330. MR:1303646 (96b:60203)

6. D. S. Budkov and S. Ya. Makhno, Functional iterated logarithm law for a Wiener process, Theory Stoch. Processes 13 (29) (2007), no. 3, 22-28. MR2396061 (2008m:60159)

7. I. I. Gikhman and A. V. Skorokhod, Stochastic Differential Equations and their Applications, Naukova dumka, Kiev, 1982. (Russian) MR678374(84j:60003)

Department of Probability Theory and Mathematical Statistics, Institute of Applied Mathematics and Mechanics, National Academy of Sciences of Ukraine, R. Luxemburg Street 74, Donetsk 83114, Ukraine

E-mail address: budkov@iamm.ac.donetsk.ua

Department of Probability Theory and Mathematical Statistics, Institute of Applied Mathematics and Mechanics, National Academy of Sciences of Ukraine, R. Luxemburg Street 74, Donetsk 83114, UKRAIne

E-mail address: makhno@iamm.ac.donetsk.ua

Received 10/DEC/2009

Originally published in Russian Translated by O. KLESOV 